\title{
Étude ethnobotanique des plantes médicinales commercialisées dans les marchés de la ville de Douala, Cameroun
}

\author{
Ladoh-Yemeda $\mathrm{CF}^{1 *}$, Vandi T², Dibong SD ${ }^{1,2}$, Mpondo Mpondo E², Wansi JD, Betti JL1', Choula F1, \\ Ndongo Din ${ }^{1}$, Tomedi Eyango $\mathrm{M}^{3}$ \\ ${ }^{1}$ Département de Biologie des Organismes Végétaux, Faculté des Sciences, Université de Douala, B.P. 24157. Douala, \\ Cameroun. \\ 2Département des Sciences Pharmaceutiques, Faculté de Médecine et des Sciences Pharmaceutiques, Université de \\ Douala, B.P. 24157. Douala Cameroun. \\ ${ }^{3}$ Département d'Aquaculture, Institut des Sciences Halieutiques, Yabassi, Cameroun. \\ *Auteur de la correspondance : christieflora@yahoo.fr
}

Original submitted in on $3^{\text {rd }}$ February 2016. Published online at www.m.elewa.org on $31^{\text {st }}$ March 2016 http://dx.doi.org/10.4314/jab.v99i1.11

\section{RÉSUMÉ}

Objectif: L'objectif de cette étude est d'inventorier et d'identifier les vertus thérapeutiques des plantes médicinales commercialisées dans la ville de Douala (Cameroun).

Méthodologie et résultats : Des enquêtes ethnobotaniques ont été conduites de juillet à août 2012, auprès de 54 herboristes dans cinq marchés en utilisant des interviews directes et semi-structurées. L'étude a permis d'identifier 84 espèces réparties en 78 genres et 50 familles dont les plus représentées ont été les Asteraceae (9 espèces), Fabaceae (6 espèces) et Lamiaceae (4 espèces). Les troubles infectieux (66,7 \%) et gynécoobstétriques et urologiques ont été majoritairement représentés. La recherche du degré de consensus (ICF) révèle que 3 catégories ont atteint des valeurs élevées : les troubles asthéniques $(0,8)$, dermatologiques $(0,6)$, gynéco-obstétriques et urologiques $(0,5)$.

Conclusion et application des résultats : Cette étude montre l'importance des enquêtes ethnobotaniques dans la phytothérapie et les limites des herboristes de la pharmacopée traditionnelle.

Mots clés : Ethnobotanique, plantes médicinales commercialisées, maladies.

\begin{abstract}
Objective: The objective of this study was to make an inventory and to identify therapeutic properties of medicinal plants sold in the city of Douala (Cameroon).

Methodology and Results: Ethnobotanical surveys were conducted from July to August 2012 beside 54 herbalists in five markets using direct and semi-structured interviews. The study identified 84 species distributed in 78 genera and 50 families, the most represented were Asteraceae ( 9 species), Fabaceae (6 species) and Lamiaceae (4 species). Infectious disorders (66.7\%) and gyneco-obstetrical and urological disorders were mostly represented. Research on the degree of consensus (ICF) reveals that three categories reached high values: asthenic (0.8), skin (0.6), gyneco-obstetrical and urological (0.5) disorders.
\end{abstract}



dans les marchés de la ville de Douala, Cameroun.

Conclusion and applications of results: This study shows the importance of ethnobotanical surveys in herbal medicine and limitations of herbalists of traditional medicine.

Keys: Ethnobotany, sold medicinal plants, diseases.

\section{INTRODUCTION}

Les plantes médicinales constituent une source importante de soins de santé dans le monde et la demande mondiale est de plus en croissante (Srivastava, 2000 ; Hamilton, 2004). La croissance démographique et l'inaccessibilité aux médicaments modernes dans les pays en développement contribuent à l'augmentation de la demande pour les médicaments traditionnels (Augustino et Gillah, 2005). En Afrique, cette demande est non seulement la résultante de l'inaccessibilité des installations modernes de soins de santé et les coûts élevés de la médecine conventionnelle mais aussi de la médecine traditionnelle qui est très souvent considérée comme une méthode de traitement plus appropriée (Marshall, 1998). Selon l'organisation mondiale de la santé, près de $80 \%$ des populations dépendent de la médecine traditionnelle pour leurs soins de santé primaire (OMS, 2002). Au Cameroun, les populations urbaines et rurales se tournent de plus en plus vers l'utilisation des plantes médicinales pour résoudre leurs problèmes de santé (Betti, 2002). Les marchés constituent une part importante de la structure du commerce des plantes médicinales sauvages dans la plupart des pays

\section{MATÉRIEL ET MÉTHODES}

Site d'étude : L'étude s'est déroulée dans la ville de Douala (latitude, $03^{\circ} 40^{\prime}-04^{\circ} 11^{\prime} \mathrm{N}$; longitude, $09^{\circ} 16^{\prime}$ $09^{\circ} 52^{\prime} \mathrm{E}$; altitude, $13 \mathrm{~m}$ ) qui a un climat appartenant au domaine équatorial d'un type particulier dit "caméronien». Ce climat se caractérise par deux saisons avec une longue saison de pluies (au moins 9 mois), des précipitations abondantes (environ $4000 \mathrm{~mm}$ par an), des températures élevées $\left(26,7^{\circ} \mathrm{C}\right)$ et stables. La moyenne minimale de température à Douala pour 30 années (1961-1990) est de $22,6^{\circ} \mathrm{C}$, en juillet et la moyenne maximale de température de $32,3^{\circ} \mathrm{C}$, en février. L'humidité relative de l'air reste élevée toute l'année et voisine de $100 \%$ (Din et al., 2008). La région du Littoral est un ensemble d'écosystèmes où se côtoient les forêts denses humides sempervirentes, notamment la forêt dense humide sempervirente littorale entre 0 et $100 \mathrm{~m}$ d'altitude et la forêt dense humide biafréenne entre africains et donnent un aperçu de la flore médicinale d'un pays (Marshall, 1998). Elles reflètent aussi bien les principales préoccupations de santé et de maladies mais également l'importance de la médecine traditionnelle pour les populations locales (Van Andel et al., 2012). L'exploitation durable des plantes médicinales commercialisées pourrait contribuer, non seulement à la préservation d'une part importante de la diversité biologique des forêts tropicales, mais également à l'amélioration des conditions de vie des communautés locales grâce à la création des revenus et au traitement des maladies (Adomou et al., 2012). L'objectif général de cette étude est d'inventorier, d'identifier les plantes médicinales commercialisées dans la ville de Douala et de recenser leurs vertus thérapeutiques afin de ressortir le profil sanitaire de la population locale. Spécifiquement, il s'agira de: (1) enquêter les herboristes de 05 marchés de la ville de Douala sur les utilisations médicinales des plantes médicinales commercialisées ; (2) identifier les plantes recensées et (3) inventorier les vertus thérapeutiques de ces plantes.

100 et $500 \mathrm{~m}$ d'altitude (Letouzey, 1985). Le choix de cette zone d'étude se justifie par le fait que la ville de Douala étant la capitale économique du Cameroun, est une ville cosmopolite où cohabitent de nombreuses tribus du Cameroun.

Enquêtes ethnobotaniques et identification des espèces médicinales : Les enquêtes ethnobotaniques ont été conduites de juillet à août 2012 dans cinq marchés de Douala en utilisant des interviews semistructurées. Ces marchés ont été retenus sur la base du nombre important des vendeurs, de leur disponibilité et du nombre de plantes médicinales vendues. II s'agit des Marchés des Chèvres, Nkolouloun, Double balle, de Ndogbassi et Dakar. Cinquante-quatre (54) herboristes indépendamment du sexe, de l'âge et de l'ethnie ont accepté de coopérer dans l'inventaire détaillé de leur(s) étal(s) de plantes médicinales. L'interview était basée sur 

dans les marchés de la ville de Douala, Cameroun.

le dialogue en langue française parfois, en langues locales accompagné parfois par l'achat d'échantillons de plantes. Les rubriques de la fiche d'enquête concernent les informations sur l'identité de l'enquêté, la connaissance et l'utilisation des plantes médicinales commercialisées. Les échantillons de plantes obtenues ont été identifiés au Laboratoire de Biologie et physiologie des Organismes Végétaux de l'Université de Douala. Les coordonnées géographiques de chaque marché ont été relevées à l'aide d'un GPS (GPSmap 62 GARMIN) d'une précision de moins de $2 \mathrm{~m}$ et le plan de localisation s'est effectué à l'aide du logiciel Arcview GIS 3.2.

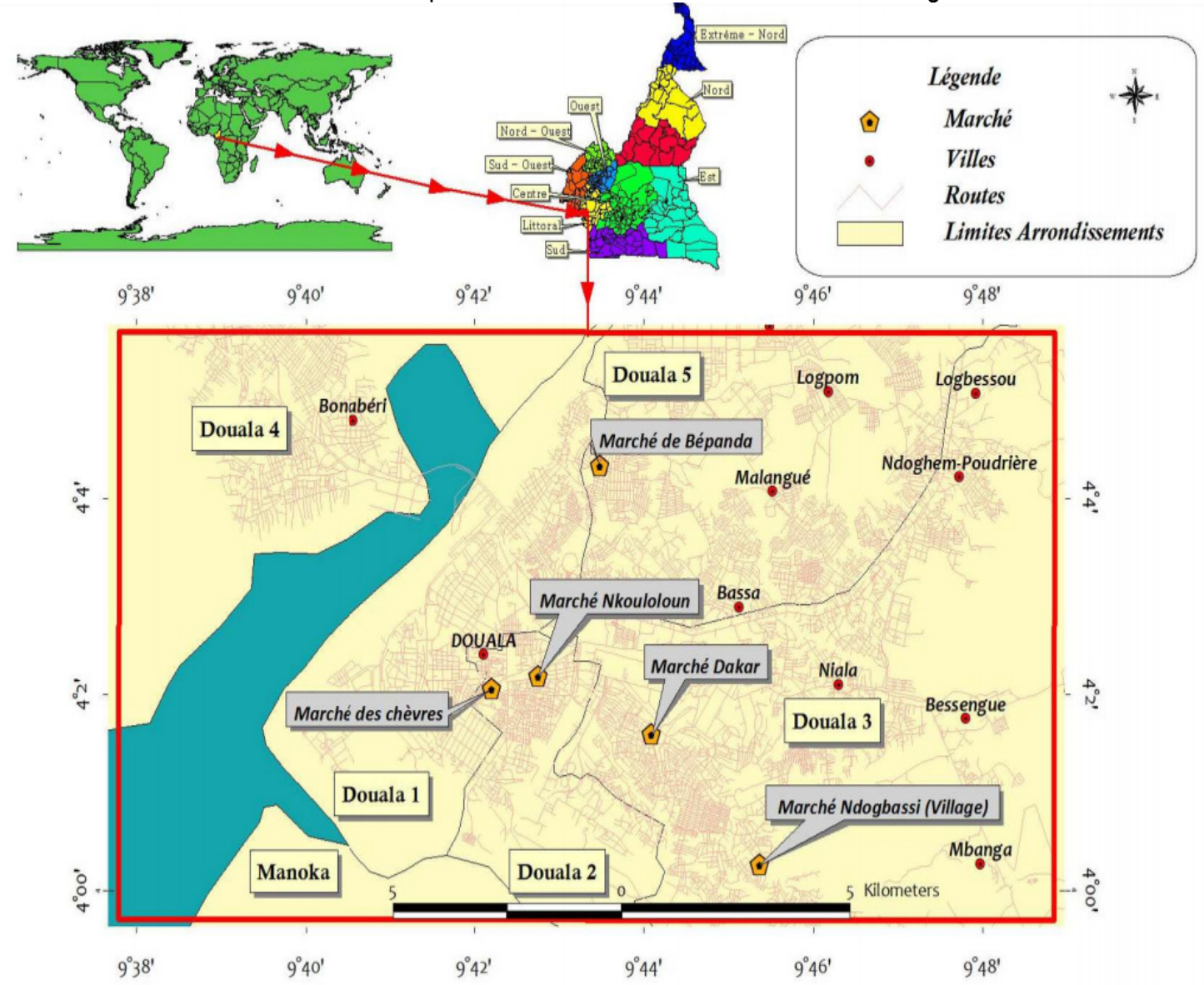

Figure 1 : Carte de localisation de la ville de Douala et des marchés enquêtés.

Analyses des données : La fréquence de citation (FC) de chaque plante commercialisée par les herboristes a été calculée suivant la formule :

$$
\mathrm{FC}=\frac{\text { Nombre de citations }}{\text { Nombre dherboristes }}
$$

Les utilisations médicinales des plantes ont été regroupées en 15 catégories: les troubles infectieux (INF), neurologiques (NEU), cardiovasculaires (CAV), respiratoires (RES), digestives et stomacales (DIS), gynéco-obstétriques et urologiques (GOU), rhumatologiques, dermatologiques (DER), ophtalmologique $(\mathrm{OPH}), \mathrm{ORL}$, endocrino-métaboliques
(ENM), infantiles (INFa), asthéniques (AST), mystiques (MYS) et autres. Pour chaque catégorie, le degré de consensus d'utilisation de la plante ou Informant Consensus Factor (ICF) a été calculé suivant la formule de Heinrich et al. (1998) :

$$
I C F=\frac{n_{u r}-n_{t}}{n_{u r}-1}
$$

Où $n_{u r}$ désigne le nombre de citations d'utilisation de chaque catégorie de maladies et $n_{t}$ le nombre total de plantes utilisées. L'ICF varie entre 0 et 1 . Une valeur faible indique que les informateurs sont en désaccord sur l'usage de la plante dans la thérapie. 


\section{RÉSULTATS}

Caractéristiques socio-démographiques des enquêtés: Pour cette étude, 54 herboristes ont été interviewers sur l'utilisation thérapeutique des plantes médicinales commercialisées dans cinq marchés de la ville de Douala (marché village, marché Dakar, marché des chèvres, marché Nkoulouloun, et marché Double balle). La majorité des herboristes est constituée des femmes soit $68,5 \%$. L'âge des herboristes a varié de 27 et 74 ans avec une majorité à plus de 50 ans $(44,4 \%)$. La région majoritaire est celle de l'Ouest $(81,5 \%)$ et de la région du Centre (11,1\%). 70,4\% admettent avoir reçu leurs savoirs de leurs ascendants, $22 \%$ reconnaissent l'avoir appris et 7,4 de manière héréditaire et empirique. $64,8 \%$ ont un niveau d'étude primaire contre $27,8 \%$ pour le niveau secondaire (Tableau 1). Seuls $9,3 \%$ des herboristes font partie d'une association de vendeurs de plantes médicinales. Les pourcentages les plus élevés des herboristes interviewés sont observés dans le marché des Chèvres (41\%) et du marché Nkoulouloun (30\%). Près de $63 \%$ des herboristes admettent commercialiser leurs plantes sur demande des clients ou des malades et sur leurs conseils également (Figure 2).

Tableau 1 : Caractéristiques socio-démographiques des herboristes interviewers $(n=54)$.

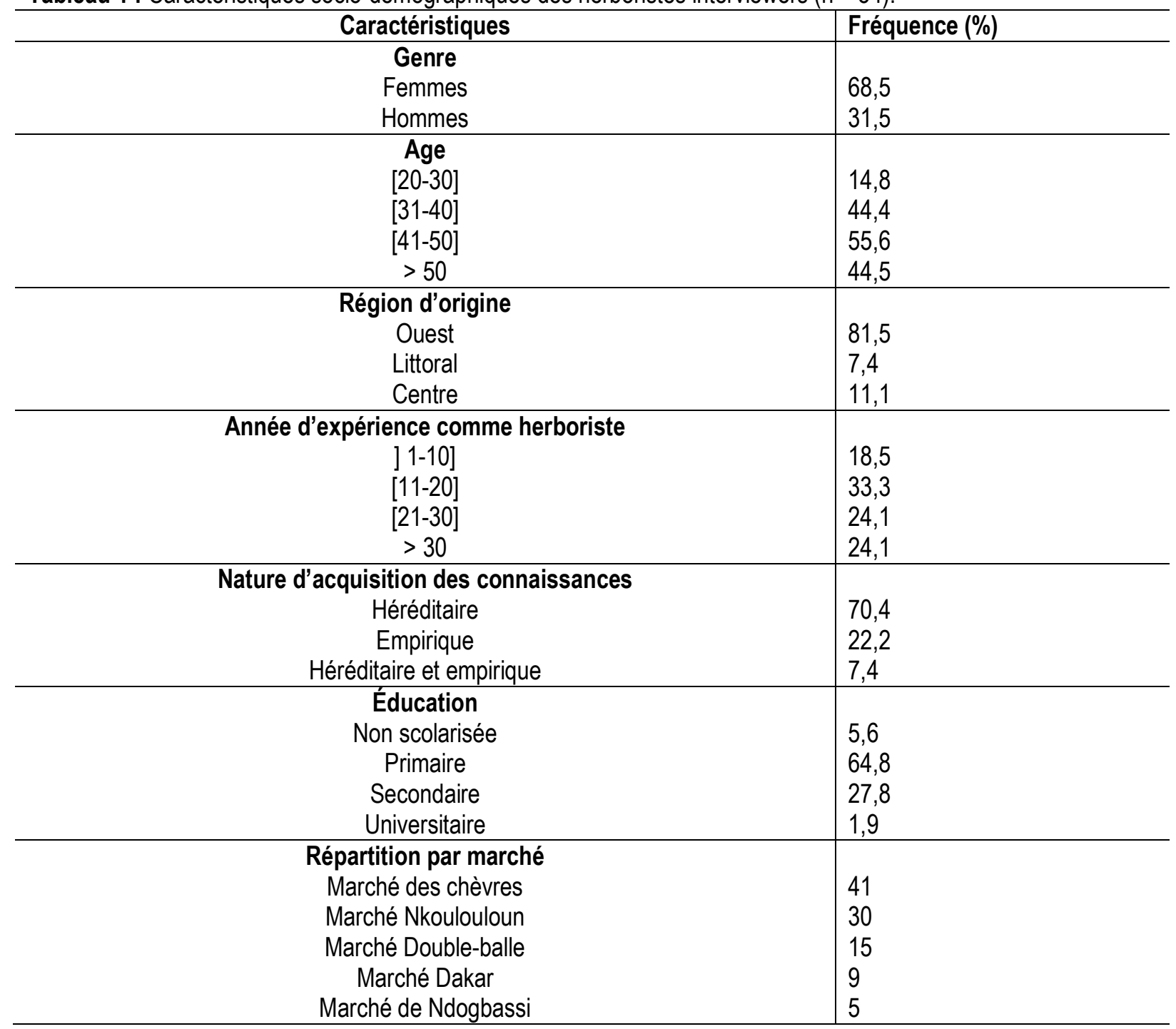



dans les marchés de la ville de Douala, Cameroun.

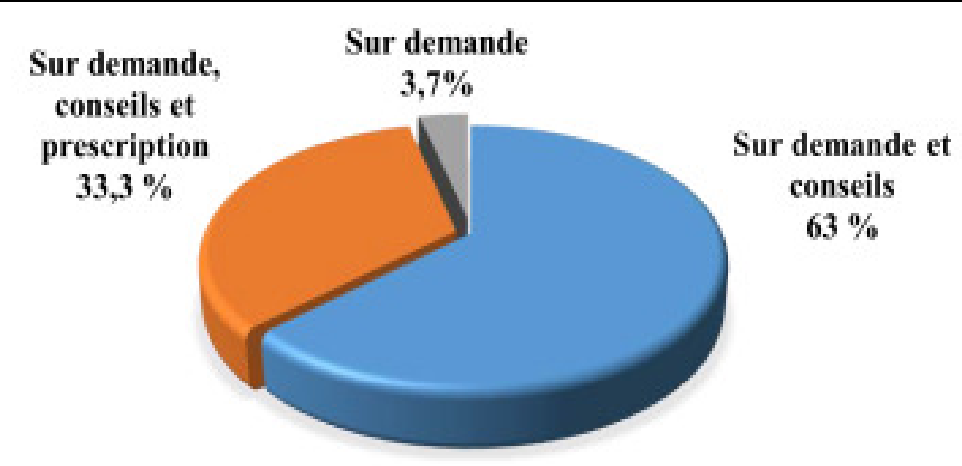

Figure 2 : Commercialisation des plantes médicinales par les herboristes.

Diversité et utilisations des plantes médicinales : $\mathrm{Au}$ total, 84 espèces de plantes médicinales commercialisées ont été identifiées dans 5 marchés de la ville de Douala (marché village, marché Dakar, marché des chèvres, marché Nkoulouloun, et marché de Bépanda double balle). Ces espèces médicinales se répartissent en 78 genres et 50 familles. Les familles les plus représentées sont les familles des Asteraceae ( 9 espèces), des Fabaceae ( 6 espèces) et des Lamiaceae (4 espèces) viennent ensuite les Acanthaceae, les Apocynaceae, Clusiaceae, les Euphorbiaceae, les Mimosaceae et les Solanaceae ( 3 espèces chacune) (Tableau 2). La plupart des plantes médicinales identifiées sont des arbres $(35,7 \%)$ suivis des arbrisseaux $(27,4 \%)$ et des herbacées $(27,4 \%)$ (Figure $3)$. Diverses parties des plantes sont commercialisées, les feuilles $(43,5 \%)$, les tiges $(22,2 \%)$ et des écorces $(21,3 \%)$ et sont majoritairement utilisées (Figure 4). Les fréquences de citation des espèces de cette étude montrent que 39 espèces ont des fréquences élevées avec des valeurs comprises entre 0,80 et 0,98 pour Cassia alata, Acmella caulirhiza, Eucalyptus arenace, Mangifera indica, Alchornea cordifolia, Citrus limon, Cymbopogon citratus, Emilia coccinea, Solanum melongena, Ageratum conyzoïdes, Aloe vera, Asystasia vogeliana, Carica papaya, Dissotis rotundifolia, Eremomastax speciosa, Ocimum basilicum, Phragmanthera capitata et Picralima nitida (Tableau 2). Aucune espèce répertoriée dans cette étude ne fait partie de la liste des espèces sauvages menacées d'extinction (CITES, 2014). La recherche du degré de consensus révèle que 3 catégories ont atteint des valeurs élevées : les troubles asthéniques $(0,8)$, dermatologiques $(0,6)$, gynéco-obstétriques et urologiques $(0,5)$, infectieuses et mystiques $(0,4)$. Cependant la fréquence d'utilisation des plantes médicinales pour le traitement des maladies montre un pourcentage élevé pour la catégorie des troubles infectieux $(66,7 \%)$ suivie des maladies gynéco- obstétriques (44 \%) et des troubles digestifs stomacaux $(3,3 \%)$. De nombreuses difficultés ont été rencontrées sur le terrain, la première a été la réticence des herboristes à donner des informations relatives à cette enquête. Certains ont demandé à être payés pour répondre aux questions et donner de leur temps. D'autres ont refusé catégoriquement de répondre parce que d'après eux les informations de l'enquête pourraient être utiliser à d'autres fins que l'étude à savoir l'édition des livres sur les plantes médicinales à leur détriment, l'ouverture de cabinets de traitement des maladies par les plantes et ce constat a été fait dans tous les marchés choisis pour cette enquête. Alors pour faire face à ces refus, la partie mode de préparation et d'administration a été supprimée de la fiche d'enquête et les herboristes semblaient plus ou moins rassurés pour la plupart. Et une somme équivalant à une bière soit 600 FCFAétait remise à chacun des herboristes ou l'achat de quelques plantes pour obtenir des réponses des herboristes. Le ravitaillement des plantes médicinales de cette étude se fait sur place dans les marchés et ces plantes sont à peu près les mêmes dans tous ces marchés. Les plantes herbacées sont en partie commercialisées par les femmes et les écorces par les hommes surtout dans les marchés des chèvres et Nkouloloum où on rencontre le plus grand nombre d'herboristes. Certains herboristes notamment les hommes en majorité, reconnaissent effectuer eux-mêmes la récolte des parties des plantes ligneuses dans les forêts environnantes et disent cependant rencontrer quelques difficultés telles que: l'interdiction de récolter certaines plantes ligneuses et les contrôles fréquents sur les routes, les saisies de leurs récoltes par les agents des eaux et des forêts, la demande de présentation d'autorisation légale d'exploitation de la forêt, l'enclavement des lieux de collecte, le mauvais état des routes et les coûts élevés de transport. 


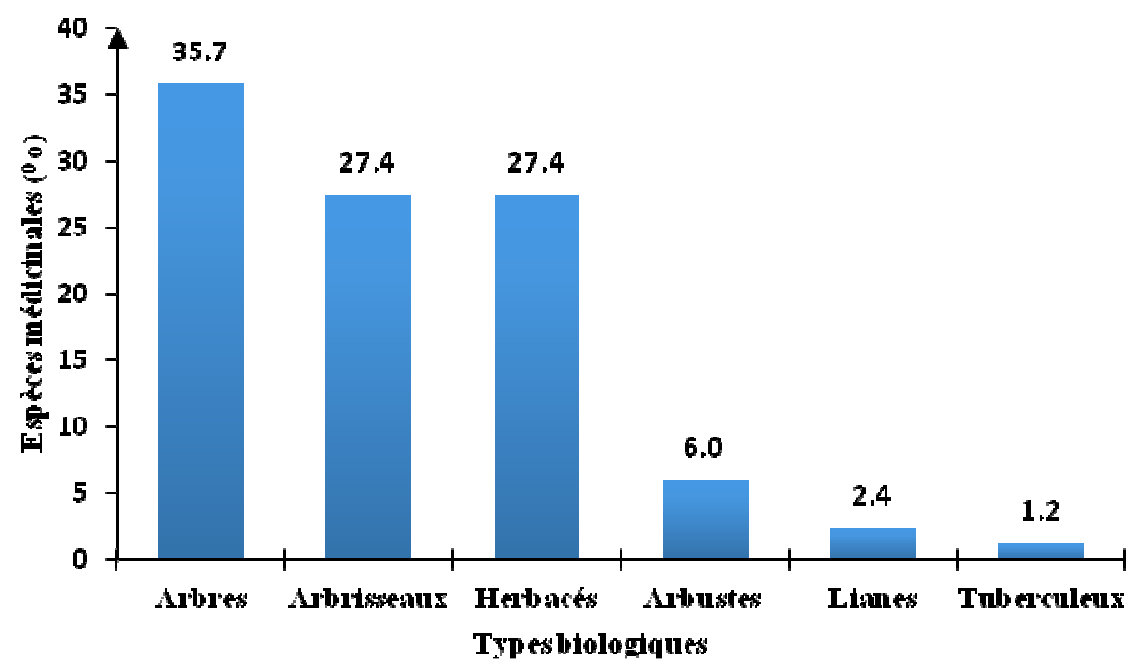

Figure 3 : Types biologiques des plantes médicinales utilisées.

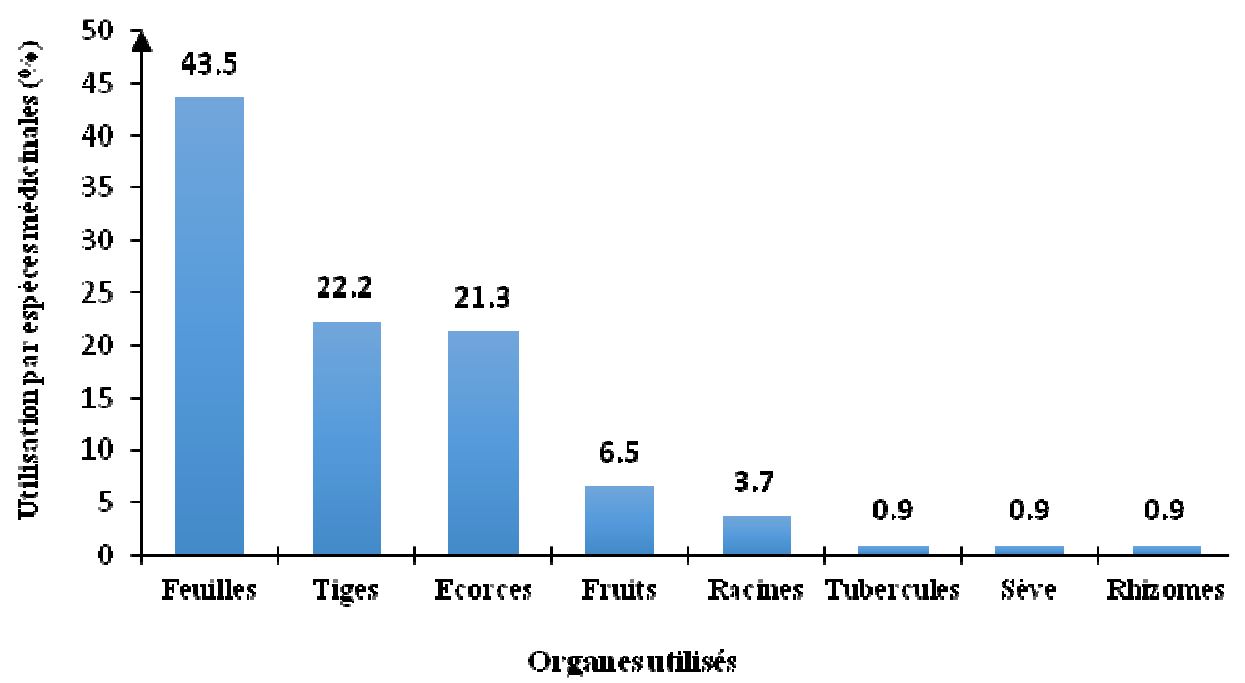

Figure 4 : Organes de plantes utilisées dans cette étude 
Ladoh-Yemeda et al. J. Appl. Biosci. 2016 Étude ethnobotanique des plantes médicinales commercialisées dans les marchés de la ville de Douala, Cameroun.

Tableau 2 : Inventaire et utilisation des plantes médicinales commercialisées dans la ville de Douala.

\begin{tabular}{|c|c|c|c|c|c|c|}
\hline NOM SCIENTIFIQUE & FAMILLE & NOM USUEL & \begin{tabular}{|l|} 
TYPE \\
BIOLOGIQUE
\end{tabular} & $\begin{array}{l}\text { PARTIES } \\
\text { UTILISEES }\end{array}$ & FC & MALADIES \\
\hline Abies alba Mill. & Pinaceae & Sapin & Arbre & Feuilles, tiges & 0,50 & INF, NEU \\
\hline $\begin{array}{l}\text { Acanthus montanus (Nees) T. } \\
\text { Anderson }\end{array}$ & Acanthacceae & Chien dent & Arbrisseau & Feuille, tiges & 0,72 & DIS \\
\hline $\begin{array}{l}\text { Acmella caulirhiza (Wall. ex DC.) } \\
\text { R.K.Jansen }\end{array}$ & Asteraceae & Yeux de la poule & Herbacée & Feuilles, tiges & 0,81 & INFa, INF, DIS, RES, EMP \\
\hline Adansonia digitata $\mathrm{L}$. & Bombacaceae & Baobab & Arbre & Ecorces & 0,31 & GYO, DIS, INF, HEM \\
\hline Afzelia bipindensis Harms & Fabaceae & $\begin{array}{l}\text { Bokeng (kentek) } \\
\text { poison de nuit }\end{array}$ & Arbre & Écorce, & 0,17 & INF, MYS, GYO, NEU \\
\hline Ageratum conyzoïdes L. & Asteraceae & Roi des herbes & Herbacée & Feuilles, tiges & 0,87 & $\begin{array}{l}\text { INF, GYO, ENM, DIS, MYS, } \\
\text { EMP }\end{array}$ \\
\hline Alafia multiflora (Stapf) Stapf & Apocynaceae & $\begin{array}{l}\text { Nyamamoto (Duala), } \\
\text { Titi-mouth (Beti) }\end{array}$ & Arbre & Ecorces & 0,13 & ENM, CAV, RHU, INF, EMP \\
\hline $\begin{array}{l}\text { Alchornea cordifolia } \\
\text { (Schumach. \& Thonn.) Müll.Arg }\end{array}$ & Euphorbiaceae & $\begin{array}{l}\text { Dibobonji (duala), } \\
\text { Ngontsi (bami) }\end{array}$ & Arbuste & Feuilles & 0,83 & HEM, DIS, AST \\
\hline Allium sp. L. & Liliaceae & Petit oignon & Herbacée & Feuilles & 0,09 & RHU, GYO, INF \\
\hline Aloe vera (L.) Burm. F. & Aloecaceae & Aloe vera & Herbacée & Feuilles & 0,87 & INF, DIS, DER, ENM \\
\hline Alstonia boobei & Apocynaceae & Kokmot (quinine) & Arbre & Écorce & 0,09 & ENM, INF, NEU, CAV, GYO \\
\hline $\begin{array}{l}\text { Annickia chlorantha (Oliv.) Setten \& } \\
\text { Maas }\end{array}$ & Annonaceae & Écorce jaune & Arbre & Ecorces & 0,70 & INF, ENM, DER, HEM, GYO \\
\hline Annona muricata L. & Annonaceae & Corossolier & Arbre & Feuilles, écorces & 0,70 & NEU \\
\hline Anthocleista vogelli Planch. & Loganiaceae & $\begin{array}{l}\text { Bolopolo (Duala) } \\
\text { babam (Bassa) }\end{array}$ & Arbre & Ecorces & 0,11 & GYO, DIS, INF, ENM, INFa, \\
\hline Asystasia vogeliana Benth. & Acanthacceae & Vert de sang & Arbrisseau & Feuilles, tiges & 0,87 & HEM, AST, INF \\
\hline Baillonella toxisperma Pierre & Sapotaceae & Moabi & Arbre & Ecorces & 0,31 & RHU, GYO, INF \\
\hline Bidens pilosa L. & Asteraceae & $\begin{array}{l}\text { Injection sauvage } \\
\text { (pupille des pauvres) }\end{array}$ & Herbacée & Feuilles, tiges & 0,70 & GYO, RHU, INF, DIS, RES \\
\hline Capsicum frutescens $L$. & Solanaceae & Petit piment & Arbrisseau & Fruit & 0,35 & INFa, DIS, INF, RHU \\
\hline Carica papaya L. & Caricaceae & Papayer & Arbre & Feuilles, racines & 0,87 & INF, DIS \\
\hline Cassia alata L. & Fabaceae & Dartier & Arbrisseau & Feuilles & 0,80 & INF, DIS, DER, RHU \\
\hline $\begin{array}{l}\text { Chromolaena odorata (L.) R. M. } \\
\text { King \& H. Rob }\end{array}$ & Asteraceae & $\begin{array}{l}\text { Bakassi (nyim), } \\
\text { woûwoû (Bami) }\end{array}$ & Arbrisseau & Feuilles, tiges & 0,22 & INF, DIS \\
\hline Cinchona pubescens Vahl & Rubiaceae & Nivaquine & Arbre & Ecorces & 0,44 & INF, ENM, CAV \\
\hline Cissus sp. L. & Vitaceae & Corde qui gratte, cinq & Liane & Tiges & 0,76 & GYO, EMP \\
\hline
\end{tabular}


Ladoh-Yemeda et al. J. Appl. Biosci. 2016 Étude ethnobotanique des plantes médicinales commercialisées dans les marchés de la ville de Douala, Cameroun.

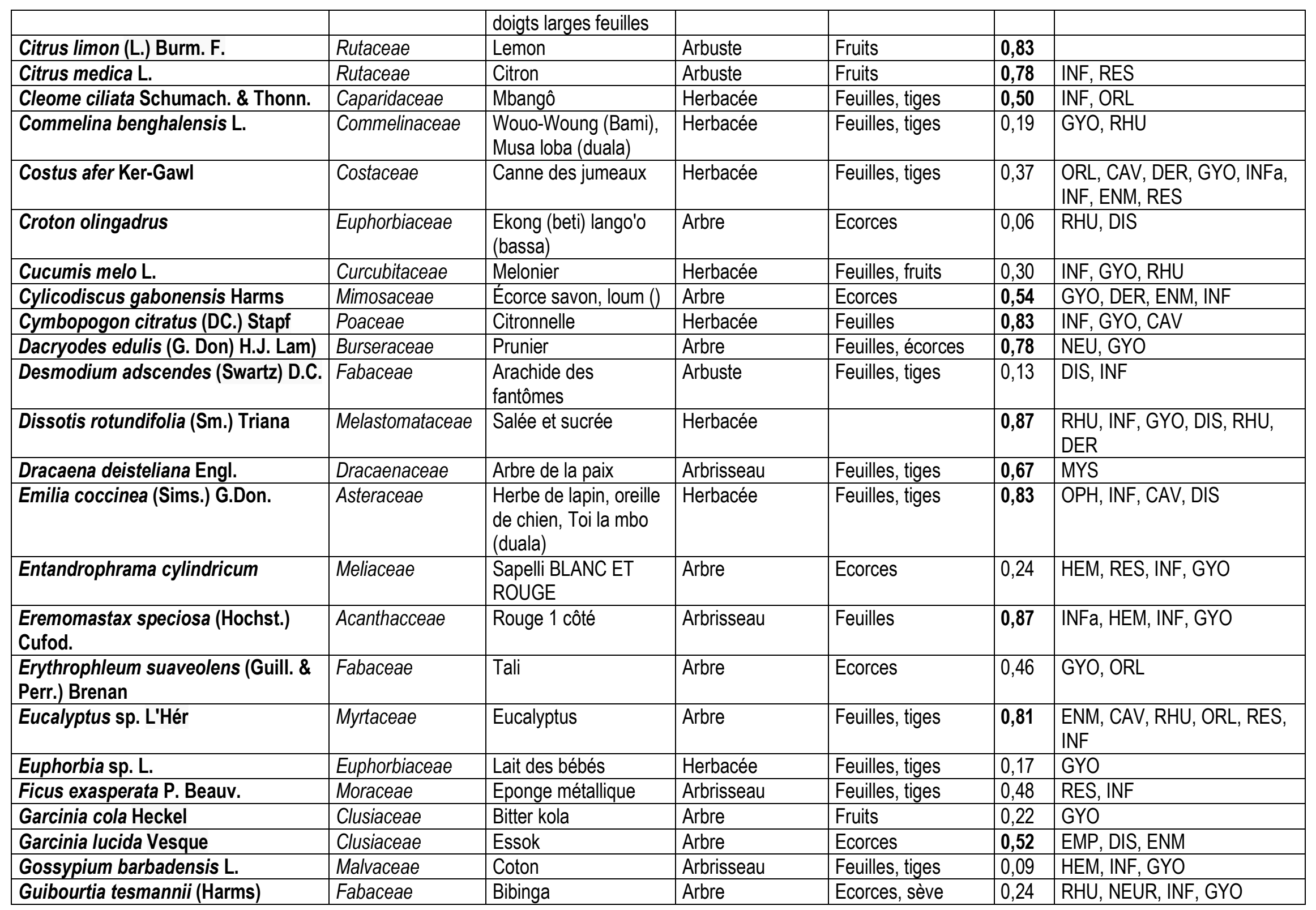


Ladoh-Yemeda et al. J. Appl. Biosci. 2016 Étude ethnobotanique des plantes médicinales commercialisées dans les marchés de la ville de Douala, Cameroun.

\begin{tabular}{|c|c|c|c|c|c|c|}
\hline Léonard & & & & & & \\
\hline Hibiscus rosa sinensis L. & Malvaceae & Hibiscus & Arbrisseau & Feuilles, tiges & 0,69 & GYO, ENM, OPH \\
\hline Ipomea irvingia & Convolvulaceae & Patate rampante & Herbacée & Feuilles, tiges & 0,17 & INF \\
\hline Ipomea sp. L. & Convolvulaceae & Patate fantôme & Herbacée & Feuilles, tiges & 0,17 & INF, GYO \\
\hline Lantana camara L. & Verbenaceae & Mbodja satan (duala) & Arbrisseau & Feuilles & 0,19 & INF, DER, GYO \\
\hline $\begin{array}{l}\text { Laportea ovalifolia (Schumach. \& } \\
\text { Thonn.) Chew }\end{array}$ & Urticaceae & Tolobanji & Arbrisseau & Feuilles, tiges & 0,17 & INFa \\
\hline Leucathemum sp. & Asteraceae & Fleur marguerite & Arbrisseau & Feuilles & 0,61 & INF, GYO \\
\hline Mammea africana $\mathrm{L}$. & Clusiaceae & $\begin{array}{l}\text { Abozô (abozock) hon } \\
\text { (bassa) }\end{array}$ & Arbre & Ecorces & 0,17 & DER, GYO, INF \\
\hline Mangifera indica L. & Anacardiaceae & Manguier & Arbre & $\begin{array}{l}\text { Feuilles, écorces, } \\
\text { racines }\end{array}$ & 0,81 & INF, GYO, DIS, INFa \\
\hline Mimosa pudica L. & Mimosaceae & $\begin{array}{l}\text { Bébé dort, Muko iyo } \\
\text { musadi (duala) }\end{array}$ & Herbacée & Feuilles & 0,28 & INF, GYO, HEM \\
\hline Musa sapientum L. & Musaceae & Bananier plantain & Herbacée & Feuilles & 0,44 & INF, DIS \\
\hline Ocimum basilicum $\mathrm{L}$. & Lamiaceae & Massep & Arbrisseau & Feuilles & 0,87 & $\begin{array}{l}\text { ORL, RHU, INF, HEM, GYO, } \\
\text { INFa, DIS }\end{array}$ \\
\hline Ocimum canum Sims. & Lamiaceae & Kotimandjo & Arbrisseau & Feuilles, tiges & 0,28 & GYO \\
\hline Panax ginseng C.A. Mey. & Araliaceae & Ginseng & Herbacée & Racines & 0,56 & ENM, CAV, INF \\
\hline Passiflora foetida L. & Passifloraceae & Bonbon mademoiselle & Herbacée & Feuilles, tiges & 0,72 & GYO, INF, DER \\
\hline Persea africana Mill. & Lauraceae & Avocatier & Arbre & Feuilles, écorces & 0,78 & CAV, NEU \\
\hline $\begin{array}{l}\text { Petersianthus macrocarpus (P. } \\
\text { Beauv.) Liben }\end{array}$ & Lecythidaceae & Abing & Arbre & Ecorces & 0,02 & INF, GYO \\
\hline Phragmanthera capitata & Loranthaceae & Samacopé & Arbrisseau & Feuilles, tiges & 0,82 & NEU, CAV, ENM \\
\hline $\begin{array}{l}\text { Picralima nitida (Stapf) T. Durand \& } \\
\text { H. Durand }\end{array}$ & Apocynaceae & $\begin{array}{l}\text { Kinquiliba, ngombak } \\
\text { (Bassa) }\end{array}$ & Arbuste & Feuilles, fruits & 0,98 & DIS, INF, ENM \\
\hline $\begin{array}{l}\text { Piptadeniastrum africanum (Hook. } \\
\text { F.) Brenan }\end{array}$ & Mimosaceae & $\begin{array}{l}\text { Dabéma, Bokombolo } \\
\text { (duala), tol (Bassa), } \\
\text { Atui (beti) }\end{array}$ & Arbre & Ecorces & 0,20 & RHU, ORL, RES, NEU \\
\hline Platosma africanum & Lamiaceae & $\begin{array}{l}\text { Ewuda bie (duala), } \\
\text { paracétamol }\end{array}$ & Arbrisseau & Feuilles, tiges & 0,63 & INFa, ENM, INF \\
\hline
\end{tabular}


Ladoh-Yemeda et al. J. Appl. Biosci. 2016 Étude ethnobotanique des plantes médicinales commercialisées dans les marchés de la ville de Douala, Cameroun.

\begin{tabular}{|c|c|c|c|c|c|c|}
\hline & & nyukutu (duala) & & & & \\
\hline Psidium guajava L. & Myrtaceae & Goyavier & Arbre & Feuilles, racines & 0,78 & DIS, INF \\
\hline $\begin{array}{l}\text { Pycnanthus angolensis (Welw.) } \\
\text { Warb. }\end{array}$ & Myristicaceae & $\begin{array}{l}\text { Carabote, Bokonda } \\
\text { (duala) Teng (Bassa), } \\
\text { Eteng (Beti) } \\
\end{array}$ & Arbre & Ecorces & 0,04 & GYO \\
\hline Saccoglottis gabonensis (Baill.) Urb. & Humiriaceae & $\begin{array}{l}\text { Bidou (Beti), Lilouhiè } \\
\text { (Bassa) }\end{array}$ & Arbre & Ecorces & 0,02 & HEM, RHU \\
\hline Selaginella myosurus (Sw.) Alston & Selaginellaceae & Tunda & Arbrisseau & Feuilles, tiges & 0,15 & AST, MYS, GYO \\
\hline Solanum melongena L. & Solanaceae & Aubergine sauvage & Arbrisseau & Feuilles, fruits & 0,85 & RHU, INF, DIS, GYO, DER \\
\hline Solanum sp. L. & Solanaceae & Sigui & Arbrisseau & Fruits & 0,04 & INF \\
\hline $\begin{array}{l}\text { Solenostemon monostachyus (P } \\
\text { Beauv.) Briq. }\end{array}$ & Lamiaceae & Dimayn (Duala) & Arbrisseau & Feuilles, tiges & 0,46 & INFa \\
\hline Stevia rebaudiana (Bertoni) Bertoni & Asteraceae & Jujube en herbe & Arbrisseau & Feuilles, tiges & 0,56 & GYO \\
\hline Tectonia grandis L. F. & Verbenaceae & Kentek & Arbre & Ecorces & 0,13 & INF \\
\hline $\begin{array}{l}\text { Tetrapleura tetraptera (Schumach. \& } \\
\text { Thonn) Taub. }\end{array}$ & Fabaceae & 4 côtés, Esesè (duala) & Liane & Feuilles, tiges & 0,46 & INFa, RHU \\
\hline Vernonia amygdalina Delile & Asteraceae & Ndolè sucré & Arbrisseau & Feuilles, tiges & 0,11 & INF \\
\hline Xanthosoma sp. Schott & Araceae & $\begin{array}{l}\text { Macabo sauvage, } \\
\text { mère des macabos }\end{array}$ & Tuberculeux & Tubercules & 0,02 & $\mathrm{RHU}$ \\
\hline Zingiber sp. Mill. & Zingiberaceae & Ndjindja rouge & Herbacée & Rhizomes & 0,04 & INF, DIS \\
\hline
\end{tabular}


Tableau 3 : Maladies citées par les herboristes et classées en catégories

\begin{tabular}{|c|c|c|c|}
\hline $\begin{array}{l}\text { Catégories de } \\
\text { maladies traitées }\end{array}$ & Affections concernées & $\begin{array}{l}\text { Nombre } \\
\text { d'espèces } \\
(n=84)\end{array}$ & ICF \\
\hline Infectieuses & $\begin{array}{l}\text { Chlamydia, antiseptique, paludisme, typhoïde, abcès, antibiotique, maladies vénériennes, infections, } \\
\text { chlamydia, chaude-pisse, blennorragie, jaunisse, dysenterie, levures, méningite, infections pulmonaires, } \\
\text { tétanos, mal de ventre, tuberculose, hépatite, tuberculose }\end{array}$ & 66,7 & 0,41 \\
\hline Neurologiques & Épilepsie, mal de nerfs, paralysie nerveuse, zona & 13,1 & 0,17 \\
\hline Cardiovasculaires & hypertension, hypotension, palpitation & 15,5 & 0,08 \\
\hline Respiratoires & Toux, infections pulmonaires & 13,1 & 0,29 \\
\hline $\begin{array}{l}\text { Digestives et } \\
\text { stomacales }\end{array}$ & $\begin{array}{l}\text { mal de dents, jaunisse, diarrhée, glaire, hémorroïdes, facilite la digestion, maux de ventre, lavage de la } \\
\text { bouche de bébés, purge des bébés, nettoyage du ventre des bébés, mauvaise haleine, bile, mal } \\
\text { gastrique, nettoyage du ventre, sirop des bébés, vomissement }\end{array}$ & 39,3 & 0,26 \\
\hline $\begin{array}{l}\text { Gynéco-obstétrique } \\
\text { et urologique }\end{array}$ & $\begin{array}{l}\text { accouchement difficile, allaitement, blennorragie, chaude-pisse, chlamydia, conception, démangeaisons } \\
\text { du pénis, démangeaisons vaginales, dilatation du col de l'utérus, eaux dans les trompes, eaux sales des } \\
\text { ventres de femmes, empêche l'avortement, faiblesses sexuelle, femmes qui viennent d'accoucher, } \\
\text { fibromes, fourmillement vaginal, gonflement des pieds des femmes enceintes, kystes, lait maternel, } \\
\text { lavage des seins de femmes, lavage vaginal, Maux de bas ventre des femmes, maladies vénériennes, } \\
\text { MST, myome, nettoyage des femmes qui viennent d'accoucher, purification du liquide amniotique, règles } \\
\text { douloureuses, règles en caillot, Règles irrégulières, stérilité, toilettes intimes, trompes bouchées, } \\
\text { varicelle, vers de femmes }\end{array}$ & 44,0 & 0,54 \\
\hline Hématologique & Anémie, leucémie & 10,7 & 0,20 \\
\hline Rhumatisme & $\begin{array}{l}\text { articulations des bébés, crampes, mal de reins, rhumatisme, douleurs lombaires, mal de dos, maux } \\
\text { articulations, du bassin, courbatures, nettoyage de la ceinture rénale }\end{array}$ & 21,4 & 0,47 \\
\hline Dermatologiques & $\begin{array}{l}\text { Acné, démangeaisons corporelles, filaires, rougeole, varicelle, mycose, teigne, dartre, varicelle, lavage } \\
\text { corporel, démangeaisons, nettoyage de la peau, blessures inguérissables, œdèmes }\end{array}$ & 9,5 & 0,56 \\
\hline Ophtalmologiques & Mal d'yeux & 2,4 & 0,00 \\
\hline O.R.L & Grippe, mal d'oreilles, sinusite, oreilles qui coulent & 9,5 & 0,00 \\
\hline Mystiques & $\begin{array}{l}\text { chasse les mauvais esprits, lavage de la malchance, bain spirituel, mauvais rêves, contre vampire, } \\
\text { couche de nuit, poison de nuit }\end{array}$ & 9,5 & 0,36 \\
\hline $\begin{array}{l}\text { Endocrino- } \\
\text { métabolique }\end{array}$ & Diabète & 19,0 & 0,00 \\
\hline Infantile & Enfants qui ne marchent pas, rate des bébés, fontanelle, rouge fesses, pour laver la bouche des bébés & 14,3 & 0,00 \\
\hline Empoisonnement & Poison & 6,0 & 0,00 \\
\hline Asthénique & fatigue générale, vitamine, fer & 2,4 & 0,75 \\
\hline
\end{tabular}




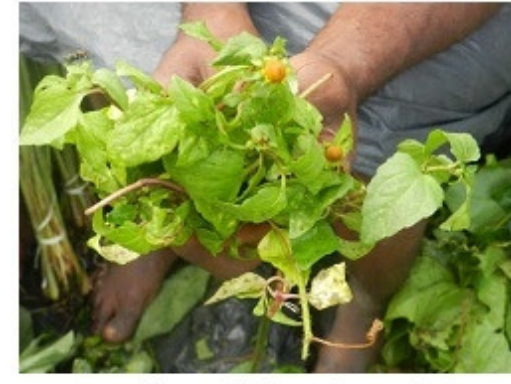

Acmella caulirhiza (Yeux de la poule)

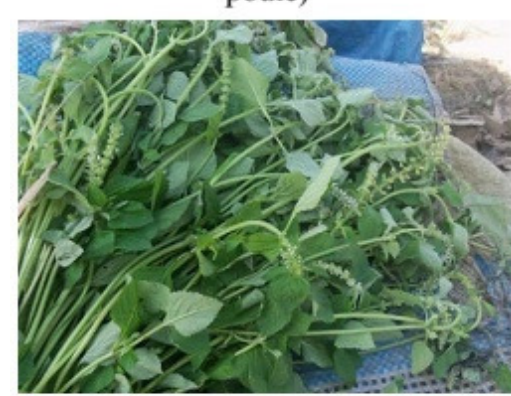

Ewuda biè (Platosma africanum)

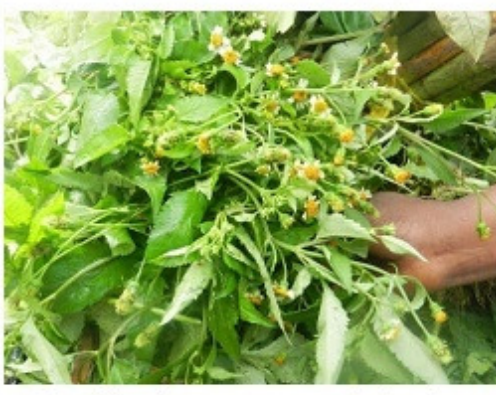

Pupilles des pauvres ou injection sauvage (Bidens pilosa)

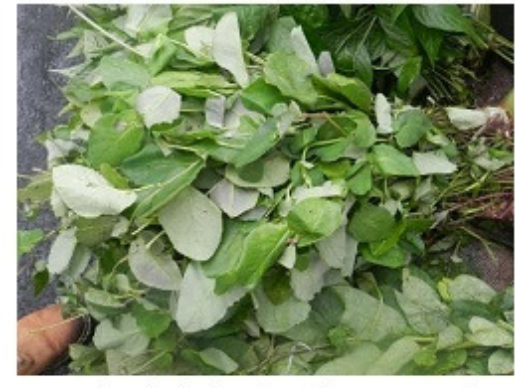

Herbe de lapin (Emilia coccinea)

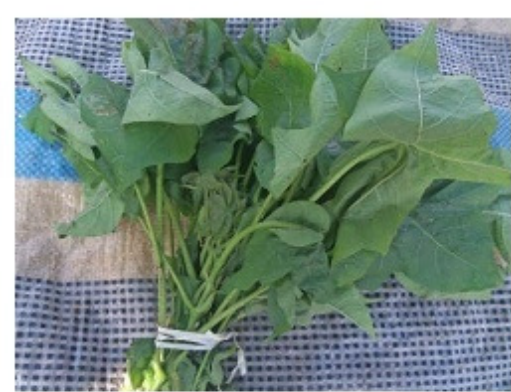

Feuilles de coton (Gossypium barbadensis)

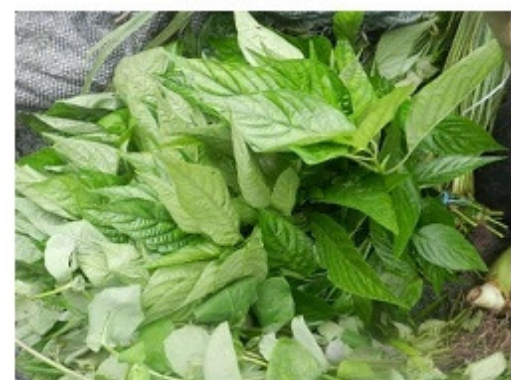

Vert de sang (Asystasia vogeliana)

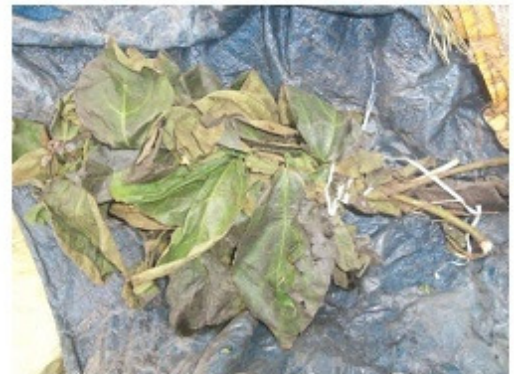

Eponge métallique (Ficus exasperata)

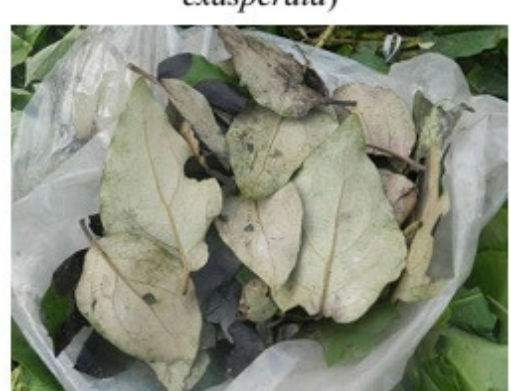

Feuilles de samacopée (Phragmanthera capitata)

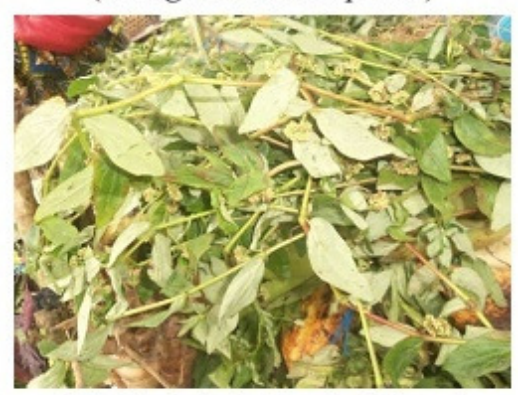

Lait des bébés (Euphorbia $\mathrm{sp}$.)

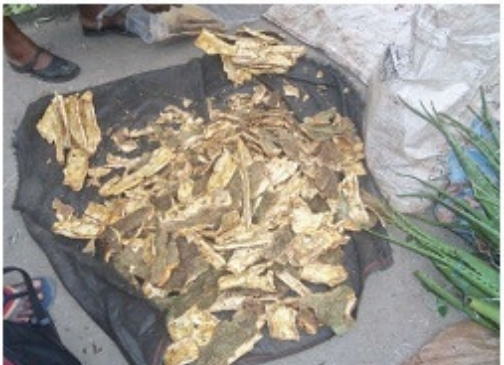

Ecorce d'Essok (Garcinia lucida)

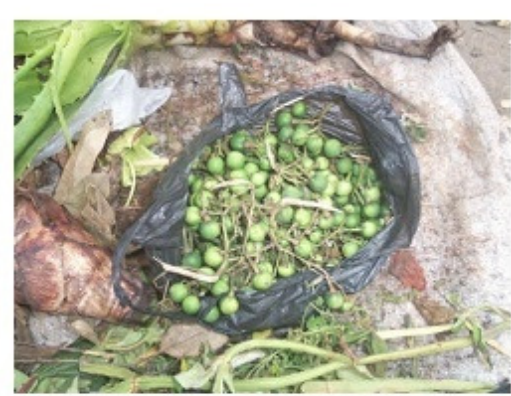

Fruits de Sigui (Solanum sp.)

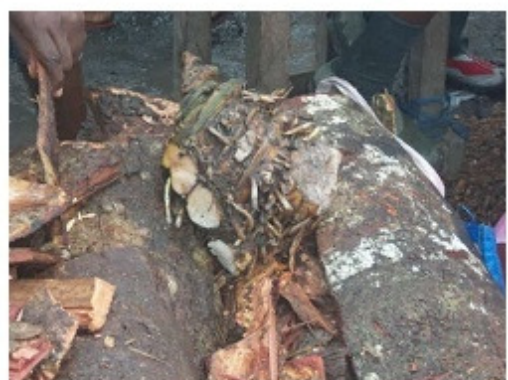

Macabo sauvage (Xanthosoma sp.)

Figure 5 : Quelques images des plantes médicinales dans les marchés enquêtés 


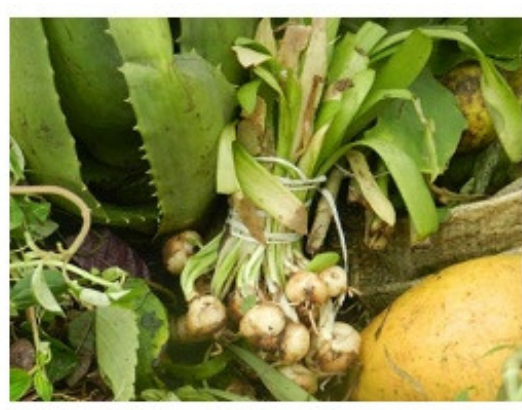

Oignon des montagnes (Allium sp.)

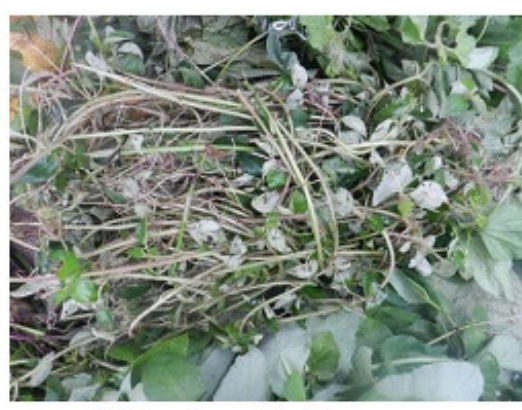

Salée et sucrée (Dracaena deisteliana

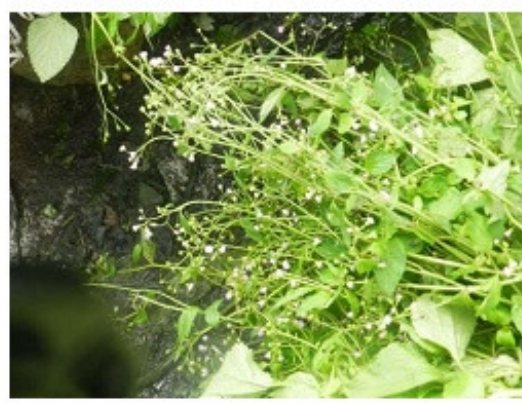

Ndolè sucré (Vernonia amygdalina)

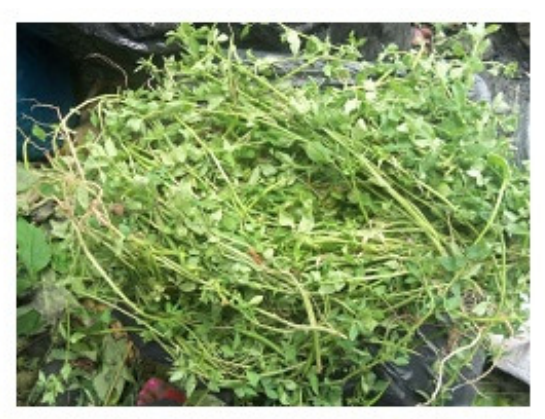

Mbango (Cleome ciliata)

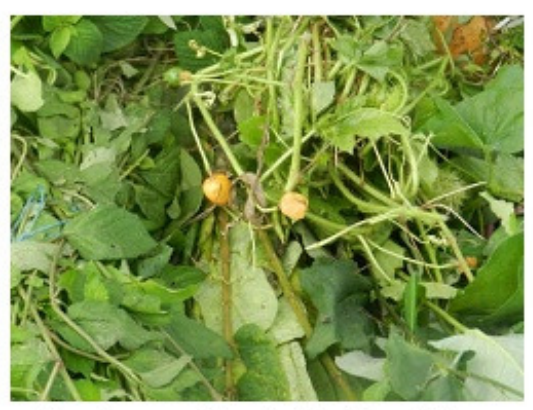

Bonbon madmoiselle (Passiflora foetida)

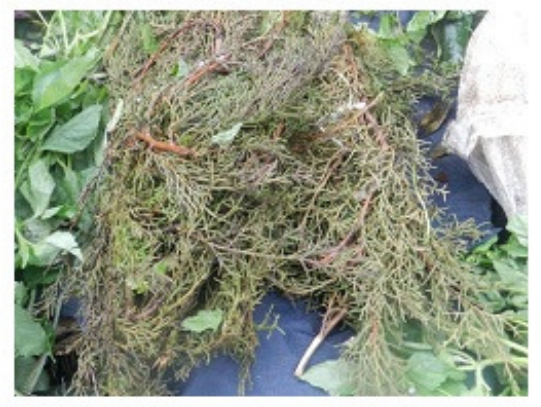

Sapin (Abies alba)

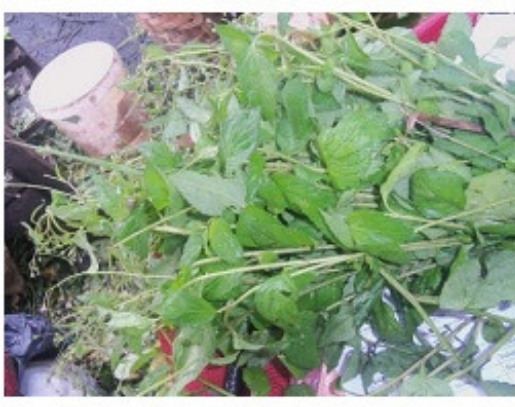

Muwiso musadi (Vernonia stellulifera)

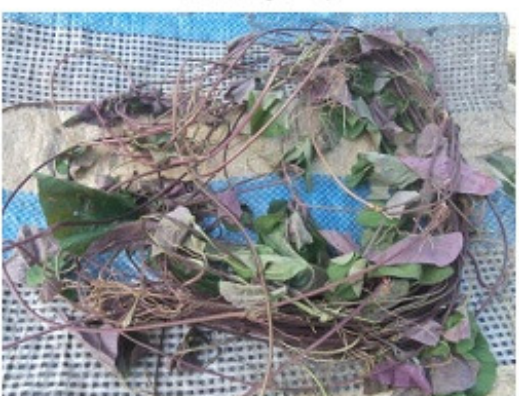

Patate fantôme (Ipomea sp.)

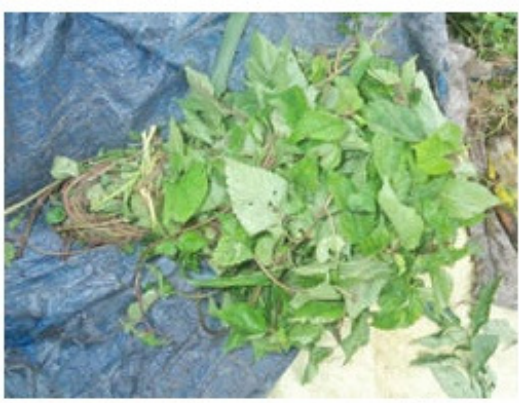

Tolobandji (Laportea ovalifolia)

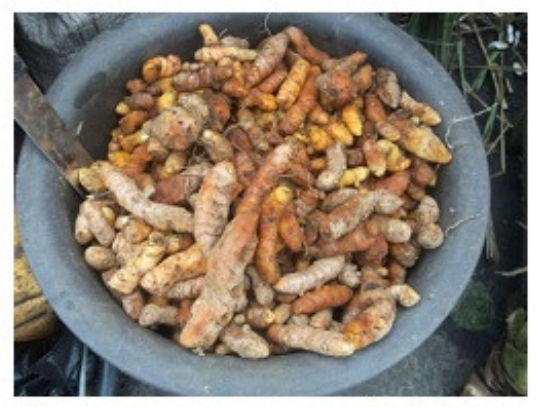

Ndjindja rouge (Zingiber sp.)

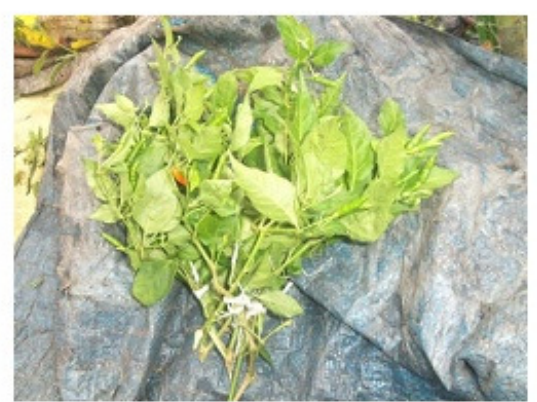

Petit piment (Capsicum frutescens)

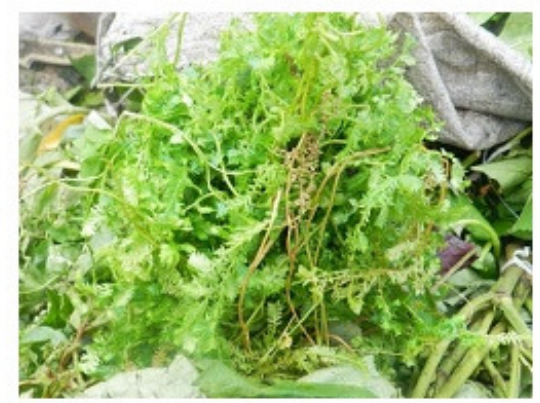

Tunda (Selaginella myosurus)

Figure 5 : Quelques plantes médicinales identifiées au cours de l'étude ethnobotanique (suite). 

dans les marchés de la ville de Douala, Cameroun.

\section{DISCUSSION}

L'étude des plantes médicinales commercialisées a révélé 84 espèces appartenant à 78 genres et 50 familles. De nombreuses études ont été conduites au Cameroun et dans d'autres pays en Afrique sur les plantes médicinales commercialisées. Au Cameroun, Dibong et al. (2011a, 2011b) identifient respectivement 30 espèces ( 25 familles et 29 genres) et 35 espèces ( 33 genres et 27 familles) dans 03 trois marchés (Nkoulouloun, Dakar et des chèvres) de la ville de Douala; Betti et al. (2002) recensent 35 espèces (35 genres et 18 familles) dans 05 marchés de la ville de Yaoundé; Au Nigeria, Idu et al. (2010) recensent 60 espèces (56 genres et 31 familles). Au Botswana, Setshogo et Mbereki (2010) inventorient 47 espèces (45 genres et 29 familles). Trabi et al. (2008) inventorient 58 espèces ( 56 genres et 33 familles) dans 3 communes de la ville d'Abidjan en Côte d'Ivoire. Le nombre d'espèces inventoriées est donc important et complète les travaux antérieurs déjà effectué dans les marchés de la ville. Les familles les plus représentées ont été les Asteraceae, Fabaceae et Lamiaceae. Au Cameroun, Ngene et al. (2015) a révélé une prédominance des Fabaceae et des Asteraceae. Dans d'autres régions africaines, la famille des Asteraceae en Ouganda par Hamil (2003), Kamatenesi-Mugisha et Oryem-Origa (2007) et en Afrique du Sud par Fennel et al. (2004) tandis que Leitao et al. (2013) obtiennent une prédominance des Asteraceae et des Lamiaceae au Brésil. II ressort de cette étude que les plantes les plus utilisées par les herboristes pour soigner les différents maux sont Cassia alata, Acmella caulirhiza, Eucalyptus arenace, Mangifera indica, Alchornea cordifolia, Citrus limon, Cymbopogon citratus, Emilia coccinea, Solanum melongena, Ageratum conyzoïdes, Aloe vera, Asystasia vogeliana, Carica papaya, Dissotis rotundifolia, Eremomastax speciosa, Ocimum basilicum, Phragmanthera capitata et Picralima nitida. Certaines de ces plantes sont revenues dans de nombreuses études ethnobotaniques antérieures. En regroupant les plantes médicinales recensées dans cette étude en plantes ligneuses et non ligneuses, une prédominance des plantes ligneuses soit $64,3 \%$ attestent ainsi leur forte utilisation dans la pharmacopée traditionnelle. Ces résultats sont en accord avec les travaux de Betti (2007) qui a révélé également l'importance thérapeutique des formes ligneuses par rapport aux formes herbacées. Les feuilles sont les organes les plus utilisées pour le traitement des maladies, suivies des tiges et des écorces. La prédominance des feuilles a été également observée par
Trabi et al., 2008. L'utilisation fréquente des feuilles serait justifiée par l'abondance des groupes chimiques qu'elles contiennent, car connues comme le lieu de synthèse des métabolites secondaires du végétal (Lumbu et al., 2005 ; Mangambu et al., 2008; Kumar et Lalramnghinglova, 2011). L'étude ethnobotanique des plantes commercialisées a permis d'inventorier 139 maladies pour lesquelles les plantes médicinales identifiées sont utilisées pour résoudre les problèmes de santé. Les maladies infectieuses prévalent $(66 \%)$ avec une ICF < 0,5. Par contre, Dibong et al. (2011a) identifient 32 maladies, idem pour Betti (2002) qui a recensé 37 maladies qu'il a regroupée en 12 catégories avec 21,9\% de citations, pour les maladies du système digestif. Ces résultats traduisent le profil sanitaire des populations de la ville de Douala fortement dominée par les maladies infectieuses et gynéco-obstétriques et urologiques. Les plantes médicinales commercialisées sont fonction de la demande (des maladies fréquentes) et de la disponibilité de ces plantes qui est souvent fonction des saisons et de la proximité avec la forêt. Au Cameroun, la majeure partie de la population n'a pas accès aux soins de santé moderne (Mpondo et al., 2012). Cette étude traduit également l'importance des plantes médicinales pour les populations locales dans la résolution des problèmes de santé primaire (Ngene et al., 2015). Les noms vernaculaires des plantes médicinales commercialisées dans cette étude sont le plus souvent en langue Duala, Bassa, Béti et quelques fois en langue Bamiléké malgré que cette dernière tribu soit la plus représentée dans cette étude. Cette contradiction serait due à l'influence de la langue parlée dans la zone, à l'origine ethnique des herboristes et aussi l'origine des lieux de récolte des plantes. Douala est une ville carrefour ayant reçu des cultures allogènes qui influencent fortement les cultures locales incluant les traditions et les pratiques médicinales traditionnelles. Les adultes se désintéressent peu ou pas à leur (s) culture (s) et se tournent davantage vers la pharmacopée populaire, à la faveur de la mondialisation. Dans cette région, les plantes ont été utilisées comme source de médicaments avant la colonisation. Les plantes devraient avoir différents usages dans des régions différentes aussi bien que dans les sites différents d'une même région (Yesilada, 2005). Le ravitaillement des plantes médicinales se fait sur place dans les marchés et ces plantes sont à peu près les mêmes dans tous ces marchés. Les plantes herbacées sont en partie commercialisées par les femmes et les écorces par les hommes, surtout dans les marchés des chèvres et 

dans les marchés de la ville de Douala, Cameroun.

Nkoulouloum où on rencontre le plus grand nombre d'herboristes. Certains herboristes notamment les hommes en majorité, reconnaissent effectuer eux-mêmes la récolte des parties des plantes ligneuses dans les forêts environnantes et disent cependant rencontrer quelques difficultés telles que : l'interdiction de récolter certaines plantes ligneuses et les contrôles fréquents sur les routes, les saisies de leurs récoltes par les agents des eaux et des forêts, la demande de présentation d'autorisation légale d'exploitation de la forêt, l'enclavement des lieux de collecte, le mauvais état des routes et les coûts élevés de transport. Le recours aux plantes médicinales par les populations locales en Afrique et dans le monde et la prévalence des maladies poussent les chercheurs à explorer cette voie, pour la découverte de nouvelles molécules biactives et la valorisation des produits de la pharmacopée traditionnelle. De nombreuses recherches sur les activités biologiques des extraits et des composés isolés ont déjà été effectuées sur les plantes médicinales recensées dans cette étude. La valorisation des plantes médicinales utilisées en médecine traditionnelle passe par leur validation scientifique pour justifier leurs utilisations. Parmi les espèces recensées, Phragmanthera capitata (Loranthaceae) communément appelée « Samacopé » a

\section{CONCLUSION}

Cette étude constitue une base de données importante pour la valorisation des plantes médicinales et la détermination du profil de santé des populations locales de Douala. Le recours aux plantes médicinales par les populations locales en Afrique et dans le monde ainsi que la prévalence des maladies, poussent les chercheurs à explorer cette voie pour la découverte de nouvelles particulièrement retenue notre attention du fait qu'elle soit une plante parasite de nombreuses espèces ligneuses. De plus, les herboristes ont admis que les propriétés de $P$. capitata seraient dépendante des plantes hôtes telles que le caféier, le goyavier, l'avocatier, le cacaoyer et le bambou de chine. Les espèces de la famille des Loranthaceae poussant sur les bambous de chine auraient des propriétés phytothérapeutiques très particulières. Cependant ces espèces sont rares. La valeur médicinale des Loranthaceae a été reconnue par 30 tradipracticiens à Lokomo, Est Cameroun (Azo'o et al., 2013). D'après ceux-ci le choix de l'espèce lors du traitement dépend de son hôte. C'est ainsi que les Loranthaceae qui poussent sur les espèces hôtes résistantes comme Irvingia gabonensis (manguier sauvage) et Mangifera indica (manguier) sont recherchées parce qu'elles sont jugées plus efficaces pour guérir les maladies. Les critères de collecte des Loranthaceae lors des traitements dépendent de la maladie à soigner ou du problème à résoudre (Azo'o et al., 2013). II serait judicieux d'évaluer et de comparer les propriétés médicinales des extraits de cette plante récoltée sur plusieurs plantes hôtes pour justifier les affirmations des herboristes dans cette étude.

molécules biactives. De nombreuses recherches sur les activités biologiques des extraits et des composés isolés ont déjà été effectuées sur les plantes médicinales recensées dans cette étude. La valorisation des plantes médicinales utilisées en médecine traditionnelle passe aussi par leur validation scientifique pour justifier leurs utilisations.

\section{REMERCIEMENTS}

Les herboristes des Marchés enquêtés dans cette étude (Marchés des Chèvres, Nkolouloun, Double balle, de Ndogbassi et Dakar enquêtés).

\section{RÉFÉRENCES BIBLIOGRAPHIQUES}

Adomou AC, Yedomonhan H, Djossa B, Legba SI, Oumorou M, Akoegninou A, 2012. Étude ethnobotanique des plantes médicinales vendues dans le marché d'Abomey-Calavi au Bénin. International Journal of Biological and Chemical Sciences 6 (2): 745-772.

Augustino S et Gillah PR, 2005. Medicinal Plants in Urban Districts of Tanzania: Plants, Gender Roles and Sustainable Use. International Forest Review 7(1): 44-58.
Azo'o JRN, Tchatat M, Mony R, Dibong SD, 2013. Parasitisme et ethnobotanique des Loranthaceae à Lokomo (Est-Cameroun). Journal of Animal and Plant Sciences 19 (2): 2923-2932.

Betti JL, 2002. Medicinal plants sold in Yaoundé markets, Cameroon. African Study Monographs 23 (2): 47-64.

Betti JL, 2007. Stratégie, plan d'action pour une meilleure collecte des données statistiques sur les produits forestiers non ligneux au Cameroun et 
recommandations pour les pays de la COMIFAC. GCP/RAF/398/GER, FAOCOMIFAC- GTZ. 154 pp.

CITES, 2014. Répertoire des espèces CITES. Programme des Nations Unies pour l'environnement. $883 \mathrm{pp}$.

Dibong SD, Mpondo Mpondo E, Ngoye A, Kwin MA, Betti JL, 2011a. Ethnobotanique et phytomédecine des plantes médicinales de Douala, Cameroun. Journal of Applied Biosciences 37: 2496-2507.

Dibong SD, Mpondo Mpondo E, Ngoye A, Priso RJ, $2011 \mathrm{~b}$. Modalities of exploitation of medicinal plants in Douala's region. American Journal of Food and Nutrition 1 (2): 67-73.

Din N, Saenger P, Priso JR, Dibong SD, Amougou A, 2008. Logging activities in mangrove forests: $A$ case study of Douala Cameroon. African Journal of Environmental Science and Technology 2 (2): 22-30.

Fennell CW, Light ME, Sparg SG, Stafford GI, van Staden J, 2004. Assessing African medicinal plants for efficacy and safety: agricultural and storage practices. Journal of Ethno pharmacology 95: 113-121.

Hamill FA, Apio S, Mubiru NK, Bukenya-Ziraba R, Mosango M, Maganyi OW, Soejarto DD, 2003. Traditional herbal drugs of Southern Uganda, II: literature analysis and antimicrobial assays. Journal of Ethnopharmacology 84: 57-78.

Hamilton AC, 2004. Medicinal plants conservation and livelihoods, biodiversity and conservation 18 (8): 1477-1517.

Heinrich M, Ankli A, Frei B, Weimann C, Sticher O, 1998. Medicinal plants in Mexico: healers consensus and cultural importance. Social Science and Medicine 47:1859-871.

Idu MacDonald, Erhabor Joseph O, Efijuemue Harriet M, 2010. Documentation on medicinal plants sold in markets in Abeokuta, Nigeria. Tropical Journal of Pharmaceutical Research 9 (2): 110-118.

Kamatenesi-Mugisha M, Oryem-Origa H, 2007. Medicinal plants used to induce labour during childbirth in western Uganda. Journal of Ethnopharmacology 109: 1-9.

Kumar $P$, Lalramnghinglova $H, 2011$. India with special reference to an Indo-Burma hotspot region. Ethnobotany, Research and Applications 9: 379420.

Leitão F, Leitão SG, Zélia de Almeida M, Cantos J, Coelho T, Da Silva PEA, 2013. Medicinal plants from open-air markets in the State of Rio de
Janeiro,Brazil as a potential source of new antimycobacterial agents. Journal of Ethnopharmacology

http://dx.doi.org/10.1016/j.jep.2013.07.009.

Letouzey R. 1985. Notice de la carte phytogéographique du Cameroun au $1: 500$ 000. 5 Vols. Toulouse, Instituts de la carte internationale de la végétation.

Lumbu S, Kahumba B, Kahambwe T, Mbayo T, Kalonda $\mathrm{M}$, Mwamba M, Penge $\mathrm{O}, 2005$. Contribution à l'étude de quelques plantes médicinales anti diarrhéiques en usage dans la ville de Lubumbashi et ses environs. Annales de Pharmacie 3(1) : 75-86.

Mangambu M, Kamabu V, Bola MF, 2008. Les plantes médicinales utilisées dans le traitement de l'asthme à Kisangani et ses environs (Province Orientale, R.D.Congo). Annales des Sciences, Université Officielle de Bukavu 1(1): 63-68.

Marshall NT, 1998. Searching for a cure: conservation of medicinal wild life resources in East and Southern Africa. Traffic International, Cambridge, United Kingdom.

Mpondo Mpondo E, Dibong SD, Ladoh Yemeda CF, Priso RJ, Ngoye A, 2012. Les plantes à phénols utilisées par les populations de la ville de Douala. Journal of Animal and Plant Sciences 15 (1): 2083-2098.

Ngene JP, Ngoule CC, Pouka Kidik CM, Mvogo Ottou PB, Ndjib RC, Dibong SD, Mpondo Mpondo E, 2015. Importance dans la pharmacopée traditionnelle des plantes à flavonoïdes vendues dans les marchés de Douala est (Cameroun). Journal of Applied Biosciences 88:8194 - 8210.

Organisation Mondiale de la Santé (OMS). 2002. Stratégie de l'OMS pour la médecine traditionnelle pour 2002-2005, Genève. 78 pp.

Setshogo Moffat P, MberekiCollen M, 2010. Floristic diversity and uses of medicinal plants sold by Street vendors in Gaborone, Botswana. African Journal of Plant Science and Biotechnology 5 (1): 69-74.

Srivastava $R, 2000$. Studying the information needs of medicinal plant stakeholders in Europe. Traffic Dispatches $15: 5$.

Trabi Fézan H, Irié Guy $M$, N'gaman Kohué CC, Clejesson HB, Mohou 2008. Études de quelques plantes thérapeutiques utilisées dans le traitement de l'hypertension artérielle et du diabète : deux maladies émergentes en Côte d'Ivoire. Sciences et Nature 5 (1): 39 - 48. 

dans les marchés de la ville de Douala, Cameroun.

Van Andel TR, Myren B, Van Onselen S, 2012. Ghana's herbal market. Journal Ethnopharmacology. http://dx.doi.org/10.1016/j.jep.2012.01.028.

Yesilada E, 2005. Past and future contribution to traditional medicine in the health care system of the Middle East. Journal of Ethnopharmacology 100 : 135-137. 